\title{
Review
}

\section{The Concept of Developing a Plasmodium vivax Malarial Vaccine with a Focus on its Pre-erythrocytic Stage}

\author{
Tuan Imaad Aarif, BSc; Sajani Dias, PhD* \\ School of Science, BMS, Colombo 06, Sri Lanka \\ "Corresponding author \\ Sajani Dias, PhD \\ Associate Dean, School of Science, BMS, 59I, Colombo 06, Sri Lanka; Tel. +94 72700 1087; E-mail: sajani@bms.edu.lk
}

\section{Article Information}

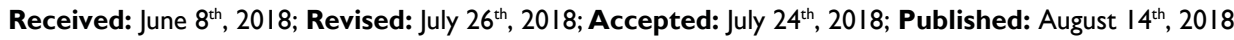

\section{Cite this article}

Aarif TI, Dias S. The concept of developing a Plasmodium vivax malarial vaccine with a focus on its pre-erythrocytic stage. Vaccin Res Open J. 20I8; 3(I): I-6. doi: 10.17I40/VROJ-3-109

\begin{abstract}
Plasmodium vivax, one of the most prevalent human malarial parasites, continues to cause significant morbidity for hundreds of millions worldwide. Due to increasing drug resistance and vivax specific relapses, immunoprophylaxis signifies a key control strategy in efforts to eradicate malaria worldwide. By far, the circumsporozoite protein (CSP) based vaccines are being developed since the 1980's. However, they have produced major limitations in eliciting partial immune responses towards the parasite. Nevertheless, the Long Synthetic Peptides (LSP's) and Vivax Malarial Protein 001 (VMP001) have produced considerable amount of immune responses and are currently undergoing phase 1 clinical trials according to several studies. Recent developments of a Virus-like particle (VLP) was found to be an efficacious vaccine candidate against a human sporozoite challenge model. The VLP's elicited high antibody titers that remained consistent for a number of days but eventually decreased. In addition, vaccine candidates such as the VLP's are currently being used to target CSP based-antigens such as the Cell-Traversal Proteins to obstruct hepatocyte invasion by sporozoites. However, substantial humoral and cellular immune responses were not produced to alleviate this process. Further research of these vaccine candidates as well as to analyze its effects towards specific CSP based antigens would immensely contribute to move clinical development forward and to further identify mechanism of immunity.
\end{abstract}

\section{Keywords}

Plasmodium vivax, Parasites, Relapses; Immunoprophylaxis; Circumsporozoite protein; Vaccine development; Immune responses; Pre-erythrocytic stage vaccine.

\section{INTRODUCTION}

$\mathrm{H}$ uman malaria is a blood infection caused by protozoal parasites of the genus Plasmodium $(\mathrm{P})$ and is transmitted by species of the Anopheles mosquitoes. It is a parasitic disease that is endemic in most tropical and subtropical regions worldwide. Four species of malarial parasites that infects humans are, $P$. falciparum, $P$. vivax, $P$. malariae and $P$. ovale. ${ }^{1}$

Plasmodium vivax, is one of the most prevalent form of the human malarial parasites, although the mortality rate is considerably lower than that for P. falciparum infection. $P$. vivax malaria causes significant morbidity for hundreds of millions of residents through- out Asia, Middle East, the Western Pacific and South America. ${ }^{2,3}$ In 2013, Plasmodium vivax alone generated a global burden of about 16 million cases annually and represents a tremendous public health problem, particularly in the South American and Asian continents. ${ }^{4,5}$

\section{List of Abbreviations}

$\begin{array}{ll}\text { WHO } & \text { - World Health Organization } \\ \text { P. vivax } & \text { - Plasmodium vivax } \\ \text { PEV } & \text { - Pre-erythrocytic vaccine } \\ \text { BSV } & \text { - Blood stage vaccine } \\ \text { TBV } & \text { - Transmission-blocking vaccines } \\ \text { CSP } & \text { - Circumsporozoite protein }\end{array}$


LSP

Alum

MTP

$\mathrm{Ab}$

ELISA

IFA

VMP001

VLP

- Long synthetic peptides

- Aluminium hydroxide

- Muramyl tripeptide

- Antibodies

- Enzyme-linked immunosorbent assay

- Indirect fluorescent antibody

- Vivax Malarial Protein 001

\section{PLASMODIUMVIVAX AND ITS LIFE CYCLE}

Plasmodium vivax parasites consists of a complex lifecycle that includes 2 cycles: (i) the sexual cycle in the female Anopheles vector, and (ii) the asexual cycle in the human host. Infection with these parasites presents an asymptomatic pre erythrocytic stage, which occurs in the liver, closely followed by a symptomatic erythrocytic stage. ${ }^{6}$

During a blood meal, when the mosquito injects its sporozoites directly into the blood stream or into the tissues of the host, the motile sporozoites penetrates small blood vessels and enters the liver. In hepatic sinusoids of the liver, they invade healthy hepatocytes. The sporozoites differentiates into mature schizonts in the hepatocytes with thousands of uninucleated merozoites surrounded by a membrane (pre-erythrocytic stage). The hepatic schizonts present in the hepatocyte rupture releasing merozoites that invade reticulocytes located in the general blood circulation. This marks the beginning of the erythrocytic stage.

Within the infected erythrocytes, the merozoite differentiates further into erythrocytic trophozoites. When the erythrocyte containing the merozoites is fully matured, it ruptures releasing its contents (merozoites) into the blood circulation, invading other erythrocytes, thereby repeating the entire erythrocytic cycle. Alternatively, some merozoites can further develop into gametocytes where during the next blood feeding, the female Anopheles mosquito species can ingest these gametocytes. This initiates the beginning of the sexual stage of the life cycle for onward transmissions. ${ }^{7,8}$

The $P$. vivax parasite is one of the most difficult species to eradicate due to its unique ability to activate its dormant liver-stage hypnozoites that resides in the liver. This activation can cause relapses of the malarial infection following its primary infection. These dormant liver-stage infection constitutes the hypnozoite reservoir of infection. This reservoir of infection can lead to various other blood infections and causes clinical attacks in different communities, providing opportunities for onward malarial transmissions. ${ }^{9}$

\section{THE STRUGGLE IN THE DEVELOPMENT OF A P. VIVAX MA- LARIAL VACCINE}

Recently the prevalence of $P$. vivax infection has increased gradually in tropical countries, indicating the development of drug resistance by the $P$. vivax strain. ${ }^{10}$ A recent study suggests that elevated regulatory $\mathrm{T}$ cells caused by anti-malarial drugs such as pyrimethamine and the combination of sulfadoxine and pyrimethamine (SP) resulted in an immune suppression in response to the $P$. vivax infection. ${ }^{10}$ Furthermore, the resistance to the an- ti-malarial drug, primaquine has caused the emergence of other $P$. vivax strains that may further increase its burden worldwide. ${ }^{11}$ Plasmodium species such as falciparum and vivax co-exists in the African continent. Therefore, the production of the highly endemic $P$. falciparum vaccine can lead to an unpredictable epidemiological outcome such as an increase in the $P$. vivax infections. Treatment for these infections (such as drugs) are present, but due to drug resistance, severity of disease, its burden and prevalence of relapses would cause a hindrance in the development of a vaccine. ${ }^{10,7}$ These concerns are of good reasons to invest and carryout research in the production of an effective $P$. vivax vaccine. Specific vaccine candidates for $P$. vivax are present and have great potential in contributing to overpowering malaria if they can be successfully developed. ${ }^{7}$

A number of potential P. vivax malaria vaccine candidates are under several stages of clinical development but their antigenic diversity among clinical isolates in different geographical areas is one of the major obstacles in the designing of an effective malarial vaccine. ${ }^{11}$

\section{DEVELOPMENT OF P. VIVAX VACCINE CANDIDATES TO- WARDS ITS LIFE CYCLE STAGES}

The complex life cycle of $P$. vivax parasite, offers a great challenge to the vaccine development process, as scientists and researchers must determine which life stage of the parasite to target as well as, to analyze whether the vaccine needs to combine several elements that target more than one life stage. Since the mid-1980s the specific focus on P. vivax vaccine development has been scarce and only a small number of $P$. vivax antigens have advanced into clinical trials. ${ }^{11}$ Since $P$. vivax comprises of three main stages of its life cycle, there are three distinct vaccine approaches being investigated towards these stages; (i) Pre-erythrocytic (PEV), (ii) blood stage (BSV), and the (iii) transmission-blocking vaccines (TBV). ${ }^{12}$ However, since the 1980's the most progressed vaccine to be developed has been made towards the pre-erythrocytic stage (PEV) of the $P$. vivax lifecycle and hence is the focus of this review. ${ }^{13,8}$

\section{PLASMODIUM VIVAX: PROGRESS TOWARDS A PRE-ERYTH- ROCYTICVACCINE}

A study done on rodent models suggested that the central repetitive domain of the Circumsporozoite protein (CSP) was present on sporozoites of rodent species that was the target of protective monoclonal antibodies. ${ }^{13}$ Further, CSP was found to be the most abundant polypeptide present on the sporozoites of all Plasmodium species that is involved in invasion into the liver hepatocytes. Over the years it has shown great potential as a target for the development of a vaccine. ${ }^{14,8}$

As opposed to P. falciparum species, a complex diversity is seen within the CSP molecule of P. vivax, where two strains of this molecule are present worldwide, VK210 and VK247, that differ in the central repetitive region of the molecule. ${ }^{12}$ The $\mathrm{N}$ and $\mathrm{C}$ terminal regions in these molecules are composed of 95 and 92 amino acids, respectively, and were shown to contain highly conserved domains which are involved in the parasite binding to host cells. ${ }^{2}$ 
Long synthetic peptides also known as LSPs corresponds to this $\mathrm{N}$ and $\mathrm{C}$ terminal regions with a third peptide (peptide $\mathrm{R}$ ) which are potential vaccine candidates as well. In addition, these LSPs contain multiple B cell, T-helper and cytotoxic $\mathrm{T}$ cell epitopes that contributes to the prevalence of antibodies to the different regions of the protein. ${ }^{1}$

\section{Vivax - I (CSP 210)}

In 1989, an initial study was done of a recombinant Plasmodium vivax vaccine known as Vivax - 1 which was derived from a plasmid transformed yeast containing the entire repeat region and part of the surrounding $\mathrm{N}$-terminal and $\mathrm{C}$-terminal regions of the CS protein (VK210). ${ }^{15}$ This vaccine was tested and coupled with another vaccine candidate known as NS181-V20 which was produced in the bacteria Escherichia coli. Forty-eight Saimiri monkeys (S. sciureus boliviensis) were administered with these two vaccine candidates along with aluminium hydroxide (alum) and muramyl tripeptide (MTP) as adjuvants. ${ }^{15}$

The vaccines administered with alum induced high levels of antibodies than the vaccines administered with MTP which were much less immunogenic. No significant difference in Ab titers was seen between the two vaccine groups receiving MTP and the group receiving neither of the adjuvants and the vaccine. According to the above study, this was the first time a P. vivax sporozoite vaccine has been tested in monkeys. ${ }^{15}$

Subsequently, another study was carried out on the same vaccine candidate Vivax-1 ( $\mathrm{rP} \nu \mathrm{CS})$ along with another purified dosage formulation ( $\mathrm{r} P$ CS-NYU) of that vaccine, which was tested in a phase I trial to assess the safety and immunogenicity in adult human volunteers, between the ages of 19 and 22 years. ${ }^{16}$ These volunteers were immunized with escalating doses of 50 , 100,200 , and $400 \mu \mathrm{g}$ of the vaccine which was initially formulated in alhydrogel. The sera from the immunized volunteers analysed by enzyme-linked immunosorbent assay (ELISA) and enzyme-linked immunosorbent assay (ELISA) showed low titers or no antibody response.

Despite three consecutive immunizations, it was concluded that this Vivax-1 vaccine candidate was poorly immunogenic in volunteers. This also indicated that the group that received the highest dose $(400 \mu \mathrm{g})$ had low or negative antibody responses. Hence, it can be concluded that other formulations or adjuvants would be required to warrant further testing of a preerythrocytic CSP vaccine. ${ }^{16}$

\section{Long Synthetic Peptides (LSP's) (CSP 210 )}

After several years, the Long Synthetic Peptides were developed which are long peptides containing sequences of the CSP molecule produced by a solid phase peptide synthesis technology. The LSP vaccine consists of three peptides, namely: the $\mathrm{N}$-terminal $(\mathrm{N})$, C-terminal (C) and the central repeat region (R) which is of more than 70 amino acids each. ${ }^{12}$ The LSP's were preferred over the
Vivax - 1 vaccine for their advantages in clinical application, such as lack of contamination with biological agents, peptide stability in the absence of proteases and rapid production of the vaccine. ${ }^{12,9}$

A study performed in 2005 recruited 69 malarial patients in Colombia to be administered three injections consisting synthetic peptides $\mathrm{N}, \mathrm{R}$, and $\mathrm{C}$, separately $(10,30$, and $100 \mu \mathrm{g}$ /dose for each peptide) which was formulated in a montanide ISA720 adjuvant. ${ }^{17}$ Peptides $\mathrm{N}$ and $\mathrm{R}$ stimulated humoral responses at all doses. However, peptide $\mathrm{C}$ stimulated humoral responses only at doses of 30 and $100 \mu \mathrm{g} /$ dose. The $\mathrm{N}$ peptide at a dose of $100 \mu \mathrm{g} / \mathrm{dose}$ elicited the greatest antibody production towards the parasite. The LSP vaccine was well tolerated and no serious adverse effects were observed.

Subsequently, a pre-clinical trial was carried out using the LSP vaccine formulated in 2005, where a total of 24 Aotus lemurinus griseimembra monkeys were vaccinated with three peptides (N, R and C) separately at a dose of $100 \mu \mathrm{g}$ formulated with either Montanide ISA 720 or Montanide ISA 51 adjuvants. ${ }^{18}$ The monkeys were immunized subcutaneously with a total volume of $500 \mu \mathrm{L}$ of the peptide formulations, three times at 0,2 and 4 months.

This trial resulted in a good reproducibility of safety, tolerability, and a greater tendency of immunogenicity for all peptides formulated in Montanide ISA 51 than in Montanide ISA 720. However, no statistically significant immune responses were observed in some individuals. ${ }^{18}$ Currently a P. vivax phase 2 trial of these CSP long synthetic peptides is being carried out to further observe efficacy of this vaccine candidate. ${ }^{2}$

\section{Vivax Malarial Protein 00I (VMP00I)}

In 2010, a vivax malarial protein 001 (VMP001) was developed as a vaccine candidate for $P$. vivax malaria. ${ }^{12}$ The vaccine candidate is a synthetic chimeric recombinant protein produced in Escherichia coli bacteria though different from its native CSP molecule. It consists of three domains; the amino $(\mathrm{N})$ terminal, carboxy $(\mathrm{C})$ terminal parts of the CSP and a short repetitive region of the immunologically diverse VK210 (Type 1) and VK247 (Type 2) strains. $^{3}$

In a study carried out in 2012, to test the stimulation of both cellular and humoral immune responses, five C57BL/6J mice per group were subcutaneously immunized with the vaccine three times (formulated in combinations of VMP001 protein with GLA-SE, R848-SE and GLA-SE/R848-SE, where GLA and R848 were the adjuvants). ${ }^{3}$ The mice were given $5 \mu \mathrm{g}$ GLA-SE or $1 \mu \mathrm{g}$ R848-SE alone as a control for this study. Blood samples were collected after 2 weeks of immunization and was tested for titers of anti-VMP001 IgG antibodies by ELISA. It should be noted that the above mentioned adjuvants contained different Toll like receptor (TLR) agonists which has the ability to stimulate immune cells such as dendritic cells (DC) to secrete cytokines, up-regulation of co-stimulatory molecule expressions and enhance antigen presentation to $\mathrm{T}$ cells. ${ }^{19}$ 
The C57BL/6J mice that were injected with VMP001 in PBS at the beginning of the 2nd immunization had low titers of anti VMP001 IgG antibodies. Whereas, after the 1st immunization with VMP001 in addition with SE, GLA-SE or R848-SE and the combination (GLA-SE/R848-SE) detected high titers of anti VMP001 IgG antibodies. Furthermore, these titers were boosted following the second and third immunizations. However, the VMP001/GLA-SE formulation elicited the highest Ab titers when compared to the other formulations.

Evidences shows that under certain conditions TLR agonists present in GLA-SE has the ability to promote suppressive as well as beneficial immune responses but nevertheless, this study supports for further clinical development of VMP001 in association with the GLA-SE adjuvant as a potential vaccine candidate for $P$. vivax malaria. ${ }^{20,3}$

\section{FURTHER RESEARCH ON PRE-ERYTHROCYTIC VACCINES}

\section{Virus-Like Particle (VLP)}

Virus-Like Particles are known to stimulate effective humoral and cellular immune responses due to their repetitive structures. The RTS,S/AS01 vaccine used to treat $P$. falciparum parasites uses a similar VLP approach which are now in phase 3 trials and are enroute to be approved by the FDA. ${ }^{21,22}$

Recent studies on CSP has developed an exceedingly protective CSP-based $P$. vivax vaccine known as VLP, that encodes the VK210 and/or VK247 PvCSP repeat regions. ${ }^{22}$ The trial tested the Rv21 efficacy with the presence of a Matrix-M adjuvant in C57BL/6 mice, a mouse strain that has previously shown to be highly sensitive to a P. berghei sporozoite infection. The mice were immunized with an Rv21 dose $(0.5 \mu \mathrm{g})$ which was followed by a challenge with 2,000 transgenic PbANKA-P $\nu$ CS VK210 sporozoites. However, this resulted in failure to show protective efficacy.

Furthermore, the dose of Rv21 (5 $\mu \mathrm{g})$ was increased the by 10 times which resulted in a complete protection in these mice even after a challenge with a higher dose of 5,000 PbANKA-P $\nu \mathrm{CS}$ VK210 sporozoites. This Rv21 immunization induced antibody titers which remained constantly high for up to 70 days.

This trial indicated that using VLP's formulated in the Matrix - M adjuvant, it can induce high levels of protective immunity even in a mouse strain that hinders a protection against a sporozoite challenge. 22

\section{Cell-Traversal Protein for Ookinetes and Sporozoites}

To develop an effective malarial vaccine, several formulations or adjuvants could be used which enables to incorporate multiple parasite antigens targeting several stages of the $P$. vivax life cycle. One recently discovered antigen representing the CSP is known as the P. vivax cell-traversal protein for ookinetes and sporozoites (PvCelTOS). ${ }^{23}$ This protein is secreted by micronemes which are important for the success of cell crossing by sporozoites and ookinetes, and also hepatocyte invasion carried out by sporozoites. Additionally, the P $\leadsto$ CelTOS is necessary for the motility of the parasite in both the mosquito vector and the human host, being the primary factor for the success of malarial infections. ${ }^{24}$

A study was carried out to an analyze of four different clinically relevant vaccine platforms to target $P \nu$ CelTOS. ${ }^{23}$ These vaccines are based on previous studies done on targeting various vaccine candidate antigens in both $P$. vivax and $P$. falciparum. One common vaccine candidate is the VLP's, derived from the bacteriophage $\mathrm{Q} \beta$ which has the ability to spontaneously assemble around bacterial RNA following expression in Escherichia coli. $^{25}$ Other vaccine candidates used in this study includes: a recombinant chimpanzee adenoviral vector (ChAd63) expressing $P \nu$ CelTOS (Ad), a recombinant MVA vector expressing P $\nu$ CelTOS (MVA) and the PrCelTOS protein produced in eukaryotic HEK293T cells (denoted as protein). The VLP's and the protein was co-administered with Matrix-M adjuvants. The main aim of this study was to evaluate the PrCelTOS-specific humoral and cellular immune responses elicited by four different immunization strategies using these platforms by a prime-boost vaccine approach, using ChAd63-P CelTOS (adenovirus [Ad]) as the priming agent.

Initially, the ChAd63-PvCelTOS (adenovirus [Ad]) was intramuscularly injected to prime immune responses in BALB/c and CD-1 mice. ${ }^{25}$ The other three vaccine platforms (Ad-MVA, Ad-protein, and Ad-VLPs) were injected intramuscularly 8 weeks later to boost responses. All vaccine platforms were injected with three groups of mice ( $\mathrm{n}=6 \mathrm{each}$ ). Following injection, blood samples were collected at 14 days after the Ad prime and at day 63 after the boosting to assess the humoral and cellular immune responses.

The resulting total $\mathrm{IgG}$ antibody levels elevated significantly after boosting with MVA, VLPs, and protein, with the highest titers being observed after boosting specifically with VLPs and protein in $\mathrm{BALB} / \mathrm{c}$ mice than in CD-1 mice (1). Subsequently, the study came to a conclusion suggesting the existence of an immunodominant $\mathrm{P} v$ CelTOS epitope for inbred BALB/c mice. Similar studies have hence been carried out to determine the value of targeting of the CelTOS in a multicomponent subunit vaccine but have failed to produce the substantial humoral and cellular immune responses. ${ }^{26,24}$

\section{CONCLUSION}

Due to the significant morbidity and recurrent incidences of Plasmodium vivax in most parts of the globe and most importantly the inability to control this infection with drugs due to drug resistance as well as the prevalence of vivax specific relapses, immunoprophylaxis represents the key control strategy, which would be influenced by the progress of a specific $P$. vivax vaccine. Studies on the parasites three life stages, have shown that the most progressed vaccines to be developed has been towards the pre-erythrocytic stage of the parasite. There are no specific vaccines for $P$. vivax that has been approved by the FDA but several CSP based vaccines were developed since the 1980's, namely: Vivax -1, Long Synthetic Peptides (LSPs) and the Vivax Malarial 
Protein 001 (VMP001). All of which have elicited high antibody titers in reference to clinical trials. However, they produced major limitations, some of which were partial immune responses and co-infections. Nevertheless, the LSP's and VMP001 are currently undergoing phase 1 clinical trials which have been distinguished as the best developed vaccines so far. The recently developed CSPbased vaccine known as a virus-like particle (VLP), 'Rv2' was found to be an efficacious vaccine candidate, eliciting antibody titers that remained consistently high for a prolong time presenting complete protection in mice. These vaccine candidates (VLP) are currently being used to target CSP based - antigens such as the CellTraversal Proteins to obstruct hepatocyte invasion by sporozoites. However, substantial humoral and cellular immune responses were not produced to alleviate this process. This indicates that future research on these vaccine candidates as well as the effects of developed vaccines against these antigens should be conducted to bring about a more potent and efficacious vaccine towards P. vivax infections.

\section{REFERENCES}

1. Herrera S, Corradin G, Arévalo-Herrera M. An update on the search for a Plasmodium vivax vaccine. Trends in Parasitology. 23(3): 122-128. 2007. doi: 10.1016/j.pt.2007.01.008

2. Corradin G, Céspedes N, Verdini A, Kajava A, Arévalo-Herrera M. et al. Malaria vaccine development using synthetic peptides as a technical platform. Synthetic Vaccines. 107-149. 2012. doi: 10.1016/B978-0-12-396548-6.00005-6

3. Lumsden J, Nurmukhambetova S, Klein J, Sattabongkot J, Bennett J, et al. Evaluation of immune responses to a Plasmodium vivax CSP-based recombinant protein vaccine candidate in combination with second-generation adjuvants in mice, Vaccine, 30(22): 3311-3319. 2012. doi: 10.1016/j.vaccine.2012.03.004

4. Lai S, Li Z, Wardrop N, Sun J, Head M., et al. Malaria in China, 2011-2015: An observational study. Bull World Health Organ. 95(8): 564-573. 2017. doi: 10.2471/BLT.17.191668

5. World Health Organization (WHO) (2018) World malaria report, Website. http://www.who.int/malaria/publications/world_ malaria_report/en/. Accessed December 122017.

6. Cornejo O, Escalante A. The origin and age of Plasmodium vivax. Trends in Parasitology. 22(12): 558-563. 2006. doi: 10.1016/j. pt.2006.09.007

7. Baer K, Klotz C, Kappe S, Schnieder T, Frevert U. Release of hepatic Plasmodium yoelii merozoites into the pulmonary microvasculature. PLoS Pathogens. 3(11): 171. 2007. doi: 10.1371/journal. ppat.0030171

8. Lima-Junior J, Pratt-Riccio L. Major histocompatibility complex and malaria: Focus on Plasmodium vivax infection. Front Immunol. 7. 2016. doi: 10.3389/fimmu.2016.00013
9. Reyes-Sandoval A, Khan S, Janse C, Bachmann M, Hill A. Development of a Plasmodium vivax malaria vaccine for clinical applications using transgenic parasites, virus-like particles and recombinant viruses. Malar J. 13: 77. 2014. doi: 10.1186/1475-287513-S1-P77

10. Chuangchaiya S, Jangpatarapongsa K., Chootong P, Sirichaisinthop J, Sattabongkot J, et al. Immune response to Plasmodium vivax has a potential to reduce malaria severity. Clinical \& Experimental Immunology. 160(2): 233-239. 2009. doi: 10.1111/j.13652249.2009.04075.x

11. Jangpatarapongsa K, Xia H, Fang Q, Hu K., Yuan Y, et al. Immunity to malaria in Plasmodium vivax infection: A Study in Central China. PLoS ONE. 7(9). 2012. doi: 10.1371/journal. pone. 0045971

12. Reyes-Sandoval A, Bachmann M. Plasmodium vivax malaria vaccines: Why are we where we are?. Hum Vaccin Immunother. 9(12): 2558-2565. 2013. doi: 10.4161/hv.26157

13. Good M, Kaslow D, Miller L. Pathways and strategies for developing a malaria blood-stage vaccine. Annu Rev Immunol. 16(1): 57-87. 1998. doi: 10.1146/annurev.immunol.16.1.57

14. Bennett J, Yadava A, Tosh D, Sattabongkot J, Komisar J, et al. Phase 1/2a trial of Plasmodium vivax malaria vaccine candidate VMP001/AS01B in malaria-naive adults: safety, immunogenicity and efficacy. PLOS Neglected Tropical Diseases. 10(2): 4423. 2016. doi: 10.1371/journal.pntd.0004423

15. Collins W, Chulay J, Barr P, Ruebush T, Bathurst I, et al. Immunization of Saimiri Sciureus Boliviensis with recombinant vaccines based on the circumsporozoite protein of Plasmodium Vivax. Am J Trop Med Hyg. 40(5): 455-464. 1989. doi: 10.4269/ ajtmh.1989.40.455

16. Herrington D, Bathurst I, Losonsky G, Hollingdale M, Levine $\mathrm{M}$, et al. Safety and immunogenicity of a recombinant sporozoite malaria vaccine against Plasmodium vivax. Am J Trop Med Hyg. 45(6): 695-701. 1991. doi: 10.1016/0264-410X(92)90047-N

17. Herrera S, Corradin G, Velez J, Soto L, Fernandez O, et al. Safety and elicitation of humoral and cellular responses in columbian malaria-naïve volunteers by a Plasmodium Vivax cicumsporozoite protein-derived synthetic vaccine. Am J Trop Med Hyg. 73: 3-9. 2005. doi: $10.4269 /$ ajtmh.2005.73.3

18. Arévalo-Herrera M, Castellanos A, Céspedes N, Soto L, Corradin $G$, et al. Preclinical vaccine study of Plasmodium vivax circumsporozoite protein derived-synthetic polypeptides formulated in Montanide ISA 720 and Montanide ISA 51 adjuvants. Am J Trop Med Hyg. 84: 21-27. 2011. doi: 10.4269/ajtmh.2011.10-0110

19. Gnjatic S, Sawhney N, Bhardwaj N. Toll-Like Receptor Agonists. The Cancer Journal. 16(4): 382-391. doi: 10.1097/ PPO.0b013e3181eaca65 
20. Yadava A, Hall C, Sullivan J, Nace D, Williams T, et al. Protective efficacy of a Plasmodium vivax circumsporozoite proteinbased vaccine in Aotus nancymaae Is associated with antibodies to the repeat region, PLoS Neglected Tropical Diseases, 8(10): .e3268. 2014. doi: 10.1371/journal.pntd.0003268

21. Souza-Neiras W, Melo L, Machado R. The genetic diversity of Plasmodium vivax: a review. Mem Inst Oswaldo Cruz: 102(3): 245254. 2007. DOI: $10.1590 /$ S0074-02762007000300002

22. Salman A, Montoya-Díaz E, West H, Lall A, Atcheson E, et al. Rational development of a protective $P$. vivax vaccine evaluated with transgenic rodent parasite challenge models. Sci Rep. 7: 46482. 2017. doi: $10.1038 /$ srep46482

23. Alves E, Salman A, Leoratti F, Lopez-Camacho C, ViverosSandoval, et al. Evaluation of Plasmodium vivax cell-traversal protein for ookinetes and sporozoites as a pre-erythrocytic $P$. vivax vaccine. Clinical and Vaccine Immunology. 24(4): 1-16. 2017. doi:

\subsection{8/cvi.00501-16}

24. Bitencourt Chaves L, Perce-da-Silva D, Rodrigues-da-Silva R., Martins da Silva J, Cassiano G, et al. Plasmodium vivax cell traversal protein for ookinetes and sporozoites (P $\nu$ CelTOS) gene sequence and potential epitopes are highly conserved among isolates from different regions of Brazilian amazon. PLOS Neglected Tropical Diseases. 11(2). 2017. doi: 10.1371/journal.pntd.0005344

25. Akache B, Weeratna R, Deora A, Thorn J, Champion, et al. Anti-IgE Qb-VLP conjugate vaccine self-adjuvants through activation of TLR7. Vaccines. 4(1): p.3. 2016. doi: 10.1371/journal. pntd.0005344

26. Rodrigues-da-Silva R., Soares I, Lopez-Camacho C, Martins da Silva J, Perce-da-Silva D, et al. Plasmodium vivax cell-traversal protein for ookinetes and sporozoites: naturally acquired humoral immune response and b-cell epitope mapping in Brazilian amazon inhabitants. Front Immunol. 8. 2017. doi: 10.3389/fimmu.2017.00077 